\title{
Los archivos audiovisuales en los medios de comunicación digital
}

\author{
Àngels Jiménez \\ $M^{a}$ Eulàlia Fuentes \\ Alfons Gonzàlez \\ Área de Documentación de la Universidad Autónoma de Barcelona
}

\subsection{Resumen}

Se ofrece una revisión, desde una perspectiva documental, del acceso a la información audiovisual en los medios digitales, haciendo un hincapié especial en la oferta de productos y servicios de información que enriquecen sus sedes web.

Palabras clave: Archivos audiovisuales. Medios de comunicación.

\subsection{Abstract}

A review of audiovisual information access in digital media is offered from an informational point of view, with an emphasis in the new products and services that are enriching their web sites.

Keywords: Audiovisual archives. Mass media.

\section{Contextualización dentro del programa de investigación}

El Área de Documentación de la Universidad Autónoma de Barcelona (UAB) tiene como uno de sus ámbitos prioritarios de investigación el estudio de la aplicación de la ciencia documental en los medios de comunicación social. Los primeros pasos del Área arrancan en los años 80 con una tesis de la doctora $\mathrm{M}^{\mathrm{a}}$ Eulàlia Fuentes dedicada a conocer el tratamiento de la documentación en los rotativos barceloneses. Tras este trabajo se han ido sucediendo otros que no han dejado de tener como objetivo el análisis de la estrecha relación entre los procesos documentales y la comunicación. Entre ellos destacan Documentación y medios de información y comunicación: análisis de la situación española frente al reto europeo, elaborado conjuntamente por $\mathrm{M}^{\mathrm{a}}$ Eulàlia Fuentes y Alicia Conesa (Conesa y Fuentes, 1994), presentado en 1992 en el 46 Congreso de la FID, y que fue el preludio del estudio publicado dos años más tarde por el Centre d'Investigació de la Comunicació de la Generalitat de Catalunya, bajo el título La Documentació periodística. Estat de la qüestió a Catalunya $i$ altres expe- 
riències europees (Fuentes y Conesa, 1994). En 1995 se publicó el Manual de Documentación periodística en el que participaron todos los miembros del área y otros expertos bajo la dirección de la doctora Fuentes.

La extensión social de Internet ha propiciado un cambio radical en la concepción de los medios de comunicación. Esta transformación no pasó desapercibida a los miembros del área y buena prueba de ello es la investigación que la doctora Fuentes presentó en 1996 con motivo de su acceso a cátedra. Este trabajo analizó las transformaciones en los servicios documentales en los medios de comunicación. A partir de ese momento se consolidó un grupo de investigación dentro del Área dedicado al estudio y análisis de las implicaciones de la documentación en la configuración del perfil de los medios digitales. En 1998 se presentó en las VI Jornadas Españolas de Documentación celebradas en Valencia una comunicación que tenía por objeto el estudio de los servicios de valor añadido en la prensa española en Internet (Fuentes y González, 1998). Un año después, en el marco de una investigación financiada por la CICYT para analizar los servicios de documentación de los medios de comunicación locales, se dedicó una atención especial tanto al uso de Internet como fuente de información por parte de estos medios, como su presencia real en la red y el nivel de sus servicios de valor añadido. Ese mismo año se presentó en las VII Jornades Catalanes de Documentació una actualización de los resultados presentados en Valencia (Jiménez, González y Fuentes, 1999).

La experiencia obtenida en esta línea de investigación permitió elaborar un capítulo en el Manual de Documentación informativa, coordinado por el doctor José Antonio Moreiro, dedicado a la documentación electrónica, en el que ocupaba un lugar destacado la gestión documental de los medios digitales (Jiménez, González y Fuentes, 2000). En el monográfico de mayo del 2000 sobre hemerotecas digitales de El profesional de la información se publicó un artículo en el que se analizaba la realidad de estos servicios de acceso a información retrospectiva a través de una extensa revisión de los principales rotativos de ámbito nacional y extranjero (Jiménez, González y Fuentes, 2000). En la edición de Ibersid del 2001 se presentó una contribución que analizó el papel del profesional de la información ante el reto de los medios de comunicación digitales, en él se hacía un hincapié especial en el caso de la prensa diaria (Jiménez, Fuentes y González, 2002). Este trabajo fue ampliado y actualizado para el Congreso Info2002 celebrado en La Habana. Una profundización en este tema dio lugar a la publicación de un artículo en el monográfico dedicado a la Documentación en los medios de comunicación de la revista Item (Jiménez, González y Fuentes, 2002).

El ámbito de interés de la línea de investigación llevada a cabo hasta la fecha se amplió para incluir en ella no sólo la realidad de la prensa digital, sino todo el abanico de servicios y sistemas de acceso a información periodística en Internet. El primer trabajo sobre este tema fue publicado en el Anuario Bibliodoc 2000

Scire. $9: 2$ (jul.-dic. 2003) 99-110. 
(Jiménez, González y Fuentes, 2001). Una de las contribuciones más significativas en esta línea investigadora la constituye la tesis de la doctora Àngels Jiménez dedicada a estudiar la gestión documental de los servicios de valor añadido de la prensa española en Internet.

La actividad investigadora se ha complementado durante estos años con la actividad docente en este mismo ámbito. La formación universitaria llevada a cabo por el Área de Documentación no se ha limitado a la Universidad Autónoma de Barcelona, sino que ha participado en cursos impartidos en otros centros como las universidades de Valencia, Murcia, El Ferrol y La Habana. Asimismo esta actividad académica también se ha orientado al terreno profesional, participando en cursos organizados por el Institut d'Educació Continua (IDEC) de Universidad Pompeu Fabra de Barcelona y la Televisión de Galicia.

\section{El escenario}

La red ha transformado el escenario de la comunicación social porque los medios de comunicación en Internet están en disposición de aprovechar las potencialidades que ofrece tanto la tecnología web como las propiedades de la información digital. Este aprovechamiento se ha traducido en una serie de características que definen el perfil de los medios digitales y los diferencian de los analógicos: interactividad, integración de información multimedia, superación de la periodicidad, inmediatez informativa, personalización de contenidos.

La prensa digital ha constituido el primer y mejor ejemplo de esta transformación y adaptación a la red. Buena parte de la explicación de esta ventaja se debe a la naturaleza textual de la información que la prensa diaria distribuye. Las limitaciones tecnológicas para la compresión y difusión de información audiovisual han supuesto un obstáculo para el acceso a este tipo de información como para el desarrollo de las sedes web de cadenas de radio y televisión. La significación profunda de estos cambios ha sido abordada por especialistas de diversos ámbitos, preferentemente sociólogos y comunicólogos. Como ya se ha puesto de manifiesto anteriormente, desde 1998 el Área de Documentación de la Facultad de Ciencias de la Comunicación de la UAB analiza la situación y evolución de los medios digitales desde una perspectiva estrictamente documental, complementaria a las que desde otras disciplinas se han elaborado.

El perfil diferenciado de los medios digitales al que antes se aludía, se ha materializado en la creación de servicios y productos informativos exclusivos del entorno digital e impensables en los medios convencionales tanto técnica como ontológicamente. Ese conjunto de servicios, resultado del aprovechamiento de un nuevo contexto tecnológico que representa Internet, lo denominamos Servicios de Documentales de Valor Añadido (SDVA). Es un término que tiene una dimen-

Scire. $9: 2$ (jul.-dic. 2003) 99-110. 
sión tautológica puesto que la idea de 'valor añadido' es inherente a la esencia de lo digital. Es una expresión que tiene validez en tanto en cuanto muestra la dirección de nuestra observación: en la exploración que nos lleva de los medios analógicos a los digitales percibimos unos cambios cuantitativos que podemos intuir también como cualitativos.

Al abordar desde una perspectiva documental en 1998 el estudio de los SDVA el primer paso fue establecer una taxonomía que permitiese distinguir entre tres tipos de servicios: servicios de acceso a información retrospectiva, servicios de acceso a información corriente, y servicios de información referencial. De la múltiple significación que los SDVA tienen, nuestro interés se centra en destacar fundamentalmente una doble contribución. Por un lado, enriquecen la capacidad informativa del medio digital. Por el otro, transforman los medios digitales en portales especializados en información de actualidad.

\section{Objetivo y metodología}

El objetivo de esta contribución ha sido conocer el nivel de acceso a la información audiovisual de actualidad proporcionada por los medios de comunicación españoles en red. Ello quiere decir que hemos dedicado una atención prioritaria a las sedes web de las televisiones tanto de alcance nacional como autonómico, pero sin perder de vista otro tipo de medios de comunicación, como la prensa, que también suele suministrar información en formato vídeo. Los datos obtenidos en esta primera aproximación a la realidad de la información audiovisual en España se han complementado con los que ha aportado la revisión de una muestra de los principales medios extranjeros.

El objeto de estudio se ha centrado en los servicios de acceso a noticias, entrevistas o reportajes de actualidad emitidos por los diferentes espacios informativos de las cadenas de televisión. Al hablar de este tipo de servicios, hacemos referencia también, por extensión, a los archivos audiovisuales. La tecnología streaming es la que posibilita la transmisión de información sonora o audiovisual de forma que el receptor no necesite almacenar en su disco duro un fichero antes de escucharlo o visualizarlo. Es decir, la información comienza a reproducirse mientras se transfiere creando un flujo continuo y constante de transmisión y ejecución. La calidad de las imágenes que se obtiene a través de esta tecnología no es comparable en la actualidad con las que ofrece la televisión convencional.

\section{Oferta informativa de las televisones españolas en Internet}

\subsection{Acceso a información corriente}

En este punto cabe distinguir entre las sedes web que tan sólo proporcionan información de actualidad en formato texto y las que lo hacen de forma audiovisual. 


\subsubsection{Suministro de información textual}

Las sedes web de todas las televisiones de ámbito nacional, a excepción de los dos canales de Radio Televisión Española (RTVE), permiten a sus usuarios acceder a información de actualidad. Se trata de noticias agrupadas en las secciones habituales de los noticiarios televisivos. La estructura y presentación de las informaciones es similar a la ofrecida por la prensa digital. RTVE suple la ausencia de este tipo de servicio mediante la posibilidad de consultar su teletexto. En el caso de las seis televisiones autonómicas tan sólo dos: la valenciana Canal 9 y la Radio Televisión Gallega no ofrecen ningún servicio de información. Cabe recordar que la televisión autonómica gallega ha sido la primera, y hasta la fecha la única en España, en emitir en tiempo real a través de Internet toda su programación.

\subsubsection{Suministro de información audiovisual}

En cuanto al acceso a información audiovisual de actualidad, la oferta se reduce sensiblemente, sobre todo entre las televisiones de alcance estatal, donde tan sólo Antena 3 y Canal + cuentan con esa posibilidad. Entre las autonómicas nuevamente las televisiones gallega y valenciana no cuentan con ningún tipo de servicio que proporcione información de actualidad.

Esta primera aproximación a las sedes web de las televisiones españolas nos permite distinguir dos conceptos bien distintos. Por un lado, aquéllas cuya finalidad parece ser simplemente la de proporcionar información corporativa, el mejor ejemplo lo constituye RTVE, ya sea a través de la descripción de los servicios de su gerencia comercial, mediante la elaboración de una revista de prensa, o dando a conocer la programación y líneas maestras de sus nuevos proyectos. En el otro lado, cabría situar a las televisiones que, sin renunciar a utilizar la red como una plataforma para consolidar su imagen corporativa, han ampliado el espectro de su oferta informativa incorporando servicios de acceso a información de actualidad. El mejor ejemplo de esta tendencia corresponde a Antena 3 que cuenta con una web específica, Antena 3 Noticias, dedicada por entero a la información de actualidad, tanto corriente como retrospectiva. Prueba de su significación es el control que la Oficina de Justificación de la Difusión (OJD) hace de ella de forma independiente a la sede web de Antena 3.

\subsection{Acceso a información retrospectiva}

Una segunda etapa en el conocimiento del estado actual de los archivos audiovisuales conduce a observar el nivel de acceso a información retrospectiva. En este sentido cabe distinguir entre las televisiones que contemplan esta posibilidad y las que de momento han renunciado a enriquecer su oferta informativa a través del diseño e implementación de un archivo de vídeos. En este último grupo se incluyen EuskalTelebista y TeleMadrid. Así pues, el número de televisiones que disponen de

Scire. $9: 2$ (jul.-dic. 2003) 99-110. 
archivos de material audiovisual con información retrospectiva se limita a cuatro. Dos son de ámbito nacional - Antena 3 y Canal +- y las otras dos son televisiones autonómicas - Televisió de Catalunya y Canal Sur de Andalucía-. Llegados a este punto y dado el reducido número televisiones con archivos audiovisuales quizá convenga reseñar de forma particular el perfil de cada uno de ellos.

1. Antena 3: El material audiovisual consultable se estructura en dos grandes bloques. Uno corresponde a las noticias de las principales secciones (nacional, internacional, deportes, etc.) y el otro a dossieres temáticos y ediciones de programas informativos ya emitidos. Cuenta con dos sistemas de consulta de sus archivos vídeo. Uno se basa en el browsing del listado de los dossieres temáticos y de las ediciones de programas, en este último caso sólo suelen contener audiovisuales relativos a las últimas cinco emisiones. El otro sistema de localización es mediante un buscador de noticias del que no se puede obtener información alguna sobre su sistema de funcionamiento, ya que no existe página de ayuda que indique cómo obtener el máximo rendimiento del buscador. Una vez efectuada una búsqueda, el documento recuperado es un texto que corresponde con la transcripción del audiovisual que se puede visualizar.

2. Canal +: Como sucede con Antena 3, la sede web de Canal + estructura la consulta de sus vídeos en dos bloques. El primero incluye noticias de actualidad clasificadas de acuerdo con las secciones periodísticas tradicionales; el segundo corresponde a dossieres temáticos. La selección del documento se efectúa a través de un sistema de browsing a partir de un listado de vídeos. Por el mismo sistema se puede consultar el archivo de los informativos de la cadena desde el 1 de julio del 2002. Para cada día desde esa fecha se puede visualizar un resumen de actualidad producido por la CNN de unos dos minutos de duración.

3. Televisió de Catalunya: Los archivos audiovisuales consultables en la sede web de la Corporació de Ràdio i Televisió de Catalunya (CRTC) son una selección de vídeos emitidos por el Telenotícies de TV3y el programa L'informatiu del Canal 33. El acceso a este tipo de documentos sólo se puede realizar a través de un buscador de noticias. La cobertura cronológica del archivo audiovisual arranca de mayo de 2001. Tal y como sucedía en el caso de Antena 3 no existe ninguna página de ayuda que muestre cómo utilizar oportunamente el buscador.

4. Canal Sur: El único sistema para acceder a la selección de documentos audiovisuales de esta cadena de televisión es a través de un buscador de noticias. Los vídeos consultables más antiguos corresponden a mayo del 2002. Tampoco en este caso existe ninguna indicación sobre el funcionamiento o características del buscador.

\section{3. Índices de programas informativos}

La documentación audiovisual de actualidad en las sedes web de las televisiones digitales en España se limita a lo comentado anteriormente. Al margen 
de esta oferta algunas televisiones han elaborado índices de programas emitidos de algunos programas especializados en reportajes de actualidad o investigación que se acompañan de sinopsis o resúmenes de los contenidos tratados. Esta información es consultable en las páginas específicas de cada programa. En el caso de RTVE destacan espacios como Documentos TV o Escarabajo verde y en Televisió de Catalunya programas especializados en reportajes de actualidad como 30 minuts i 60 minuts.

\section{Archivos audiovisuales en la prensa diaria}

Las limitaciones tecnológicas para que las ediciones digitales de la prensa incorporen información audiovisual son las mismas para las sedes web de las televisiones. Desde este punto de vista los medios de comunicación en la red tienden hacia la multimedialidad. Eso no quiere decir que la incorporación de las noticias en formato vídeo sea una realidad generalizada entre los diarios digitales, aunque sí existen experiencias interesantes. Una de ellas es la del diario El Mundo, que proporciona acceso a más de 150 vídeos y cuya cobertura cronológica se inicia en el mes de diciembre del 2001. Entre los rotativos extranjeros que han incorporado este tipo de servicios de acceso a información retrospectiva audiovisual destacan el argentino La Nación y el norteamericano The Washington Post.

La valoración que se puede hacer de la situación de los archivos audiovisuales de las televisiones españolas en Internet se puede resumir en dos puntos. Por un lado, el volumen de información audiovisual a la que dan acceso es muy discreta. No hemos de perder de vista que estamos frente a sedes web de televisiones, y de las diez cadenas españolas tan sólo una emite en directo a través de la red. Este dato es muy significativo si lo comparamos con las emisoras de radio que emiten en línea o el número de rotativos que informan a través de sus ediciones digitales. Este desequilibrio se traduce en el volumen de la información audiovisual consultable. Sólo cuatro cadenas ofrecen esa posibilidad. Es sintomático que existan diarios como El Mundo que ofrezcan un volumen de vídeos superior a cadenas de televisión como Canal + o Canal Sur. Por el otro, se detecta un nivel de aplicación muy rudimentario en los procesos básicos de la cadena documental y ello se demuestra en los sistemas de consulta y localización de la información audiovisual. Es cierto que el volumen discreto de este tipo de materiales no exige un desarrollo de complejos sistemas de clasificación, pero la inexistencia de este tipo de instrumento también dificultaría una correcta localización de los documentos si su número fuera mayor. En otros casos en los que el sistema de localización se efectúa a través de un buscador, la ausencia de una página explicativa limita el uso potencial de la información audiovisual.

Los aspectos anteriormente reseñados conducen a una consideración extradocumental. El papel más que secundario que en este tipo de sedes desempeña

Scire. $9: 2$ (jul.-dic. 2003) 99-110. 
la información audiovisual parece que también incluiría a la propia información de actualidad. Salvando alguna excepción, todo apunta a creer que en España estamos todavía lejos de contemplar a las televisiones en la red como portales de información de actualidad. Esta consideración no debe interpretarse como una crítica, sino como la constatación de un proceso gradual de maduración y perfeccionamiento que las sedes web de las televisiones en España llevarán a cabo, como hemos visto que han hecho los principales diarios digitales españoles.

\section{Rasgos distintivos de los archivos audiovisuales de las principales televisiones extranjeras}

La revisión, siempre desde un punto de vista documental, de la situación de las principales televisiones de nuestro entorno es un buen indicador para conocer la dirección que tomarán las televisiones en España

- Volumen de información: Frente al más que discreto número de documentos audiovisuales que proporcionan los archivos de las televisiones españolas, existen ejemplos significativos con un volumen de información audiovisual disponible más que considerable. Así la cadena norteamericana CBS o la francesa TF1 cuentan con archivos cuya cobertura cronológica arranca en el año 1999. La tendencia a constituir sedes web especializadas en suministrar información de actualidad se consolida en las principales televisiones de nuestro entorno al proporcionar acceso a archivos de noticias en formato texto. El caso más significativo, por el volumen de la información de su archivo, corresponde a la cadena CNN, con noticias desde 1995. Otros ejemplos los constituyen los archivos de la BBC, la italiana RAI o la norteamericana ABC. En todos estos casos el inicio de sus archivos se sitúa en el año 1997.

- Sistemas de consulta: El valor de grandes volúmenes de información sólo se hace plenamente efectivo si se diseñan sistemas de recuperación refinados que permitan una explotación selectiva de la información. En contraposición al carácter rudimentario de los instrumentos de localización de los documentos audiovisuales en las televisiones en España, la práctica totalidad de las televisiones extranjeras que cuentan con archivos de volumen considerable disponen de sistemas de consulta refinados, tal y como se muestra en la figura 1.

- Difusión de noticias por correo electrónico: Tal y como sucede en muchos diarios en la red, algunas sedes web de televisiones han puesto en marcha servicios de envío de noticias por correo electrónico. En España todavía no existe ningún ejemplo similar a los servicios de la venezolana Venevisión, los Daily e-mail o News tickers de BBC o las News alerts de ABC.

Scire. $9: 2$ (jul.-dic. 2003) 99-110. 


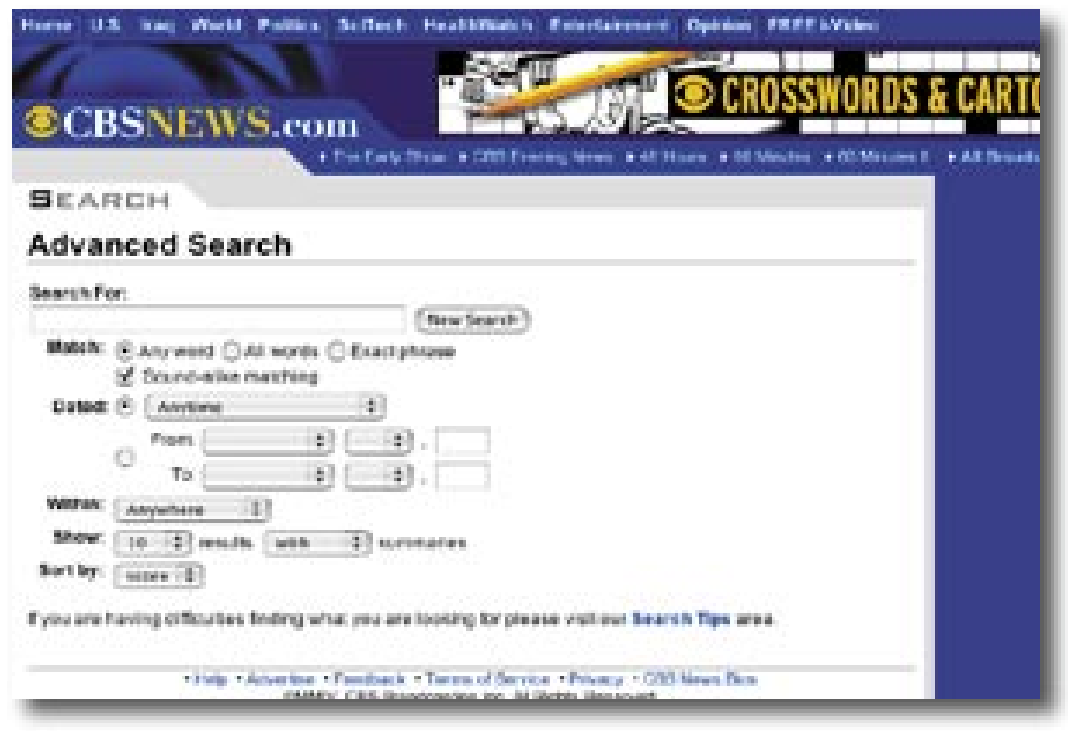

Figura 1. Sistema de consulta del archivo audiovisual de la cadena CBS

- Transcripción de entrevistas y programas informativos: Algunas televisiones, guiadas por el afán de suministrar el máximo de información sobre sus programas de actualidad, han desarrollado sistemas de consulta del contenido de tales espacios. Este el caso de la cadena norteamericana ABC.

\section{Otras iniciativas}

Una somera revisión de la realidad actual en relación con el acceso a la información audiovisual suministrada por los medios de comunicación en la red nos lleva a reseñar las siguientes iniciativas.

\subsection{Archivos audiovisuales especializados: C-SPAN}

La industria de la televisión por cable norteamericana creó en 1979 C-SPAN (Cable-Satellite Public Affairs Network, www.c-span.org) para emitir en directo la actividad parlamentaria en la Cámara de Representantes de los Estados Unidos. En 1986 C-SPAN2 surgió para dar cobertura a la actividad política en el Senado y en 1997 con C-SPAN3 hace el seguimiento del resto de actividades públicas de la clase política norteamericana. En ese mismo año C-SPAN comenzó a ofrecer información audiovisual en directo por Internet tanto del Senado como de la Cámara de Representantes. C-SPAN dispone de un archivo de vídeos con una cobertura temporal de dos semanas. Es posible consultarlo a través de dos siste- 
mas. Mediante una clasificación temática que agrupa los vídeos en diez categorías o bien, a través de un buscador. El volumen de información disponible ronda el millar de vídeos, que suponen más de 800 horas de emisión.

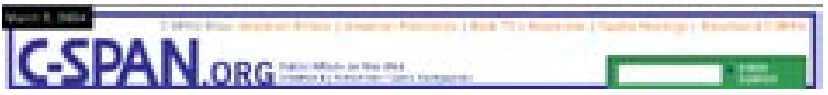

Fig. 2. Cabecera de la sede web de C-SPAN

\subsection{Archivos colectivos}

1. ITN Archive (www.itn.co.uk): Es un archivo que contiene la descripción de más de 300.000 horas de filmación. En él se incluyen tanto noticias de la cadena ITN como documentos audiovisuales procedentes de agencias de noticias de Reuters desde 1896, y de productoras de noticiarios como Universal News, Paramount British, Gaumont British, Visnews. La función de este archivo es sobre todo dar noticia detallada del contenido de cada documento, indicar su localización y facilitar su adquisición.

2. Television Archive (tvnews3.televisionarchive.org/tvarchive): Es una organización sin ánimo de lucro creada en 2001 a raíz de los sucesos del 11 de Septiembre. Su misión es la preservar las noticias emitidas por las diversas cadenas de televisión norteamericanas y proporcionar una documentación básica para el estudio de la historia reciente. El fondo accesible en la actualidad se limita a la colección de documentos audiovisuales sobre los sucesos del 11 de Septiembre. Son más de 600 horas de vídeo procedentes de emisiones de televisiones de todo el mundo.

\section{6.) TelevisionArchive}

Fig. 3. Logotipo del Television Archive

3. CBC Archives (archives.cbc.ca): Los archivos de la Canadian Broadcasting Corporation dan acceso gratuito a una selección de material audiovisual procedente tanto de sus emisiones de radio desde 1936 como de televisión desde 1952. Todo ello es el resultado de un proyecto llevado a cabo conjuntamente por la CBC y el Canadian Culture Online Program of Canadian Heritage. Se ha seleccionado una treintena de temas y acontecimientos que hayan marcado la actualidad a partir de las emisiones de radio y televisión de esta cadena canadiense. Los temas se han estructurado en dossieres formados por extractos audiovisuales y acompañados de una información contextual. La consulta se puede efectuar a través de una clasificación temática, o bien mediante un sistema de búsqueda por palabras clave.

Scire. $9: 2$ (jul.-dic. 2003) 99-110. 


\subsection{Bases de datos referenciales}

1. Television News Archive (http://tvnews.vanderbilt.edu/index.html): Es una iniciativa de la universidad norteamericana de Vanderbilt, que consiste en un importante archivo con diversas secciones en las que se recogen resúmenes textuales de los noticiarios de las cadenas ABC, $\mathrm{CBS}$ y NBC, selecciones de noticias sobre temas de especial relevancia, etc. La información que incluye, desde el 5 de agosto de 1968, puede consultarse de forma gratuita mediante diversos sistemas, incluida la búsqueda por palabras clave. La obtención de copias de vídeo de las noticias es un servicio de pago.

2. Footagenet ( $w w w$.footgenet.com): Se trata de un recurso que permite buscar noticias, reportajes, documentales, etc., en una variada selección de archivos, tanto de cadenas de televisión como de otro tipo de instituciones con importantes fondos documentales audiovisuales. La búsqueda se puede restringir a alguno de los archivos particulares o bien puede efectuarse simultáneamente en el conjunto de todos ellos. De cada uno de los resultados obtenidos se ofrece una exhaustiva descripción. Si bien la búsqueda a través de este sistema es gratuita, la adquisición de copias de los documentos debe realizarse y pagarse directamente a la institución que lo posee.

\section{Conclusión}

Desde que a inicios de la década de los años noventa la tecnología web transformó el panorama de la información electrónica, los medios de comunicación en la red han superado ya diversas etapas. En un primer momento sus sedes web respondieron a un imperativo empresarial: Representaron una estrategia más a la hora de publicitar la imagen de la empresa informativa. Posteriormente - en especial los diarios - , fueron incorporando junto a los contenidos tradicionales de la versión impresa una serie de servicios y productos que han ido enriqueciendo la función informativa del medio. En buena medida esta oferta de servicios se ha basado en la aplicación de técnicas y procesos típicamente documentales. La creación de hemerotecas virtuales y la elaboración de directorios selectivos de recursos digitales atiende perfectamente a este punto de vista. Las potencialidades de la red han permitido incluso superar el perfil tradicional de los medios impresos. En la actualidad las grandes cabeceras de la prensa nacional y extranjera han incorporado morfologías informativas que en el mundo analógico serían imposibles. Desde este punto de vista, la prensa digital parece constituir el mejor ejemplo de adaptación y aprovechamiento del entorno Internet. Frente a ese modelo de adaptación, otros medios como las televisones tienen ante sí el reto de superar los obstáculos tecnológicos para la difusión de sus contenidos audioviosuales.

Scire. $9: 2$ (jul.-dic. 2003) 99-110. 


\section{Referencias}

Conesa, Alicia; Fuentes i Pujol, M. ${ }^{a}$ Eulàlia (1994). Reference Services and mass media: analysis of the Spanish situation before the European challenge. // ÁlvarezOssorio, J.R.; Goedegebuure, B.G. New Worlds in Information and Documentation. Proceedings of the Forty-Sixth FID Conference and Congress (Madrid: 22-30 octubre 1992). Amsterdam-London-New York-Tokyo: Elsevier, 1994. 201-221.

Fuentes i Pujol, M. ${ }^{a}$ Eulàlia (ed.). (1995). Manual de Documentación Periodística. Madrid: Síntesis, 1995. 135-145.

Fuentes i Pujol, M. ${ }^{a}$ Eulàlia; González Quesada, Alfons (1998). La prensa española en Internet: análisis de los servicios de valor añadido. // Jornadas Españolas de Documentación (6enes: València: 1998). Valencia: Universitat de València, 1998. 281-292.

Fuentes i Pujol, M. ${ }^{a}$ Eulàlia; Conesa, Alícia (1994). La documentació periodística: Catalunya, Espanya i altres experiències europees. Barcelona: Centre d'Investigació de la Comunicació, Generalitat de Catalunya, 1994.

Jiménez, M. ${ }^{a}$ Àngels; González Quesada, Alfons; Fuentes i Pujol, M. ${ }^{a}$ Eulàlia (1999). Gestió documental de la informació en els serveis de valor afegit de la premsa espanyola a Internet. // Jornades Catalanes de Documentació (7es: Barcelona: 1999).

Jiménez, M. ${ }^{a}$ Àngels; González Quesada, Alfons; Fuentes, M. ${ }^{a}$ Eulàlia (2000a). Documentación e información electrónica. // José Antonio Moreiro (coord.). Manual de Documentación Periodística. Madrid: Cátedra, 2000. 344-425.

Jiménez, M. ${ }^{a}$ Àngels; González Quesada, Alfons; Fuentes i Pujol, M. ${ }^{a}$ Eulàlia (2000b). Las hemerotecas digitales de la prensa en Internet. // El Profesional de la Información. 9: 5 (2000) 15-22.

Jiménez, M. ${ }^{a}$ Àngels; González Quesada, Alfons; Fuentes i Pujol, M. ${ }^{a}$ Eulàlia (2001). Sistemas y servicios de acceso a información periodística en Internet. // Eulàlia Fuentes (dir.). Anuari de Biblioteconomia, Documentació i Informació = Anuario de Biblioteconomia, Documentación e Información $=$ Library and information sciences yearbook: Bibliodoc. Barcelona: COBDC, 2001. 51-68.

Jiménez, M. ${ }^{a}$ Àngels; González Quesada, Alfons; Fuentes i Pujol, M. ${ }^{a}$ Eulàlia (2002). Diaris digitals a Internet: panorama actual i expectatives professionals per als bibliotecaris-documentalistes. // Item. 32 (2002) 24-44.

Jiménez, M. ${ }^{a}$ Àngels; Fuentes i Pujol, M. ${ }^{a}$ Eulàlia; González Quesada, Alfons (2002). El profesional de la información ante el reto de los medios de comunicación digitales: el caso de la prensa. // Scire. 8:2 (2002) 141-152. 